\title{
Valoración integral de la condición física en estudiantes de la ESPOCH: Un diagnóstico oportuno.
}

Comprehensive assessment of physical condition in ESPOCH students: A timely diagnosis.

Luis Gustavo Díaz. ${ }^{1}$, Pablo Luis Lomas Badillo. ${ }^{2}$, Jorge Giovanny Tocto Lobato. ${ }^{3} \&$ Olguer Fabián Sánchez Espinoza. ${ }^{4}$

Recibido: 27-01-2021 / Revisado: 01-02-2021 /Aceptado: 27-02-2021/ Publicado: 05-03-2021

Abstract

DOI https://doi.org/10.33262/concienciadigital.v4i1.2.1605

Introduction. Studying the physical condition of university students from the approach of timely diagnosis, continues to be an aspect of great importance and utility, however, it is not always possible to merge all the necessary components that allow a comprehensive assessment of the physical condition of young students. Objective. To provide a system of indicators for the comprehensive assessment of the physical condition of university students, from the point of view of timely diagnosis. Methodology. The type of research was descriptive through a mixed approach, articulating quantitative and qualitative elements, accompanied by the use of theoretical and empirical methods. The sample was selected intentionally under the inclusion criteria of studying at the Higher Polytechnic School of Chimborazo (ESPOCH) in 7 faculties, being made up of 1097 students. We worked with different instruments which allowed us to make a single measurement. Results. 1. A system of indicators adapted to the characteristics and conditions of the participants is provided, which contributed to a comprehensive assessment of the physical condition of the participating students. 2. The importance and usefulness of timely diagnosis of the physical condition of the evaluated students is reaffirmed as an essential aspect for their integral development. Conclusions. The study of physical condition, carried out in a timely manner in the university student population, is very useful; It favors, through the recognition of the evaluation indicators, the establishment of

\footnotetext{
${ }^{1}$ Escuela Superior Politécnica de Chimborazo ESPOCH, gustavodiaz@espoch.edu.ec

${ }^{2}$ Escuela Superior Politécnica de Chimborazo ESPOCH, plomas@espoch.edu.ec

${ }^{3}$ Escuela Superior Politécnica de Chimborazo ESPOCH, jtocto@qespoch.edu.ec

${ }^{4}$ Gobierno Descentralizado de Mocha, fsanchez_ec@yahoo.com
} 
intervention strategies aimed at improving the quality of life of the university student population and providing viable solutions for the prevention of possible diseases and the optimization of the individual performance of each student.

Keywords: physical condition, timely diagnosis, students, ESPOCH

\section{Resumen}

Introducción. Estudiar la condición física de los estudiantes universitarios desde el enfoque del diagnóstico oportuno, sigue constituyendo un aspecto de gran importancia y utilidad, sin embargo, no siempre se logran fusionar todos los componentes necesarios que permitan realizar la valoración integral de la condición física de los jóvenes estudiantes. Objetivo. Aportar un sistema de indicadores para la valoración integral de la condición física en los jóvenes universitarios, desde el enfoque del diagnóstico oportuno. Metodología. El tipo de investigación fue descriptiva mediante un enfoque mixto, articulándose elementos cuantitativos y cualitativos, acompañada del empleo de métodos teóricos y empíricos. La muestra fue seleccionada de forma intencional bajo el criterio de inclusión de estar cursando estudios en la Escuela Superior Politécnica de Chimborazo (ESPOCH) en 7 facultades, quedando constituida por 1097 estudiantes. Se trabajó con diferentes instrumentos los cuales permitieron realizar una medición única. Resultados.

1. Se aporta un sistema de indicadores adaptados a las características y condiciones de los participantes, el cual contribuyó a realizar la valoración integral del estado de la condición física de los estudiantes participantes.2. Se reafirma la importancia y utilidad del diagnóstico oportuno sobre la condición física en los estudiantes evaluados como aspecto esencial para su desarrollo integral. Conclusiones. El estudio de la condición física, realizado de manera oportuna en la población estudiantil universitaria resulta de mucha utilidad; el mismo favorece mediante el reconocimiento de los indicadores de evaluación establecer estrategias de intervención encaminadas a mejorar la calidad de vida de la población estudiantil universitaria y aportar soluciones viables para la prevención de posibles enfermedades y la optimización del desempeño individual de cada estudiante.

Palabras claves: condición física, diagnóstico oportuno, estudiantes, ESPOCH

\section{Introducción.}

La Organización Panamericana de la Salud, (OPS), en el 2019 en su informe el Plan de Acción Mundial sobre Actividad Física 2018-2030, indica que, a nivel mundial, el 23\% de los adultos y el $81 \%$ de los adolescentes (de 11 a 17 años), no siguen las recomendaciones de la OMS sobre la práctica sistemática de la actividad física para la salud.

Según se explica en este informe, la prevalencia de inactividad varía considerablemente entre los diferentes países, llegando incluso al $80 \%$ en algunos grupos de adultos. En este informe se expone que la inactividad física en adultos es más alta en las regiones del 
Mediterráneo Oriental, las Américas, Europa y el Pacífico Occidental, y más baja en la de Asia Sudoriental. Como es de suponer todo ello tiene mucha relación con problemas relacionados con el desarrollo económico, tecnológico e incluso cultural.

En este orden de ideas resultan importantes los aportes de Arbós, (2017), al señalar que la actividad física practicada con regularidad provoca una disminución de los porcentajes de todas las causas de mortalidad, tales como: cardiopatías coronarias, hipertensión arterial, infarto, síndrome metabólico, diabetes tipo II, cáncer de mama, de colon, depresión, entre otros. Se coincide con la autora mencionada al destacar que los efectos preventivos que tiene la actividad física tanto en la salud funcional como en la aptitud cardiorrespiratoria, muscular y en la salud ósea deben ser considerados y tenidos en cuenta por la población mundial.

Es por ello que buscar alternativas que permitan perfeccionar los estilos de vida de la población desde la edad infantil hasta el adulto mayor, incluidos los estudiantes universitarios ha venido constituyendo una de las principales prioridades de todos los entes gubernamentales, educativos y sociales.

En el caso de los estudiantes universitarios, lo planteado adquiere gran jerarquía, precisamente por la estrecha relación que se da entre estilo de vida saludable, condición física, calidad de vida y formación integral. Mejorar la condición física de los jóvenes permite contribuir de manera creadora a su proceso de formación integral. Es por esto que estudiar la condición física de los universitarios desde el enfoque del diagnóstico oportuno, sigue constituyendo un aspecto esencial, sin embargo, no siempre se logran fusionar todos los componentes necesarios que permitan realizar la valoración integral de la condición física de los jóvenes estudiantes.

En este sentido se concuerda con Valdés, et al.,(2015), al señalar que debido a múltiples factores en muchas ocasiones los estudiantes universitarios durante su formación, manifiestan una mayor susceptibilidad a la hora de adquirir hábitos inadecuados de alimentación, los cuales podrían asentarse a lo largo de su vida de forma negativa, perjudicando grandemente su salud.

Martínez, (2008), explica que, dentro del enfoque de universidad saludable, se pretende contribuir a la promoción de estilos de vida más activos, lo cual implica entre otros aspectos reflexionar sobre el quehacer cotidiano, los hábitos poco saludables, la condición física y el sedentarismo como problemática de salud pública.

Para Laguado \& Gómez, (2014), llevar un estilo de vida saludable, se relaciona con la reducción de enfermedades crónicas no transmisibles. Enfatizan que es una etapa que se considera puede llevar a que las personas tomen diferentes decisiones y entre estas la adquisición de buenos hábitos, es la vida universitaria.

En este ámbito, Ruiz, et al., (2009), definen al nivel de condición física como la capacidad para realizar actividad física y/o ejercicio físico y su valoración representa una medida que especifica el estado integrado de las principales funciones orgánicas que intervienen en el movimiento corporal de las personas. 
Por otra parte, es importante comprender que el Índice de Masa Corporal (IMC) presenta una relación intrínseca con la condición física; al respecto concordamos con Zenić, et al., (2013), al apegarse a otros autores y señalar que el IMC es el predictor antropométrico más significativo del estado de la condición física.

Diversos autores, tales como Rush, et al.,(2014), han expresado que las últimas investigaciones realizadas, afirman que un índice bajo de condición física constituye uno de los factores que se asocian de manera directa con el sobrepeso y la obesidad infantojuvenil.

En esta dirección, se han desarrollado varios estudios relacionados con la interrelación existente entre los niveles de condición física y el peso corporal. Al respecto coincidimos con Gálvez, et al., (2015), quienes basados en estudios realizados han demostrado que los estudiantes que poseen niveles superiores de condición física presentan una mayor tendencia a un estatus de peso corporal dentro de parámetros normales.

Por otra parte, también se han desarrollado estudios referentes a la incidencia que tiene en los estudiantes el periodo de enseñanza universitario, dado el incremento de carga académica y el stress que la misma incorpora a los mismos. Respecto a ello, González, et al., (2017), indican en su investigación que la propia rutina universitaria que se caracteriza por el estrés, la carga académica y el escaso tiempo para la realización de actividades físicas, hacen susceptibles a los universitarios a sufrir obesidad.

Es por ello que se hace muy necesario la incorporación de programas de actividades físicas que ayuden a enfrentar esta situación que se presenta en los estudiantes universitarios. En esta dirección Vásquez-Gómez, et al., (2018), destacan que se hace imprescindible instaurar acompañamiento para el cuidado de la composición corporal, el nivel de actividad física y la condición física dentro de la universidad.

Estudios realizados por varios autores, han evidenciado divergencias en la relación actividad física y la condición física de los estudiantes, en esta dirección Guillamón, (2018), ha afirmado que es de gran importancia continuar profundizando en el análisis de la relación existente entre la Actividad física (AF) y la condición física (CF), y a su vez con la salud de escolares y adolescentes. No obstante, aunque no exista consenso sobre la relación entre AF y $\mathrm{CF}$ en los estudiantes, lo que sí resulta innegable es la necesidad de realización de actividad física de manera sistemática para tener una buena salud.

En observación realizada en la Escuela Superior Politécnica de Chimborazo (ESPOCH), resultó evidente la presencia de grupos de jóvenes procedentes de las diferentes carreras con disímiles valores en su condición física, a esto se une la carencia de un sistema de indicadores que permitan de forma oportuna mediante un proceso de diagnóstico determinar la condición física y caracterizar la población estudiantil universitaria; ante esta realidad surge la necesidad estudiar la condición física de los jóvenes que integran las diferentes carreras. De ahí que el objetivo general de la investigación se centró en: 
Aportar un sistema de indicadores para la valoración integral de la condición física en los jóvenes universitarios, desde el enfoque del diagnóstico oportuno.

\section{Metodología}

El tipo de investigación fue descriptiva mediante un enfoque mixto, articulándose elementos cuantitativos y cualitativos, acompañada del empleo de métodos teóricos, tales como: el histórico -lógico, el analítico-sintético, inductivo -deductivo y la observación como método empírico. La muestra fue seleccionada de forma intencional bajo el criterio de inclusión de estar cursando estudios en la Escuela Superior Politécnica de Chimborazo (ESPOCH) en 7 facultades, quedando constituida por 1097 estudiantes. Se trabajó con diferentes instrumentos los cuales permitieron realizar una medición única a toda la muestra.

Dentro de los indicadores determinados para el estudio de la condición física se encuentran: Edad, Peso, Talla, IMC, Resistencia, Salto Horizontal, Flexibilidad, Flexiones de Brazos, Abdominales y Velocidad. Obsérvese Fig No1.

\section{Figura No 1 - Indicadores considerados para el estudio de la Condición Física}

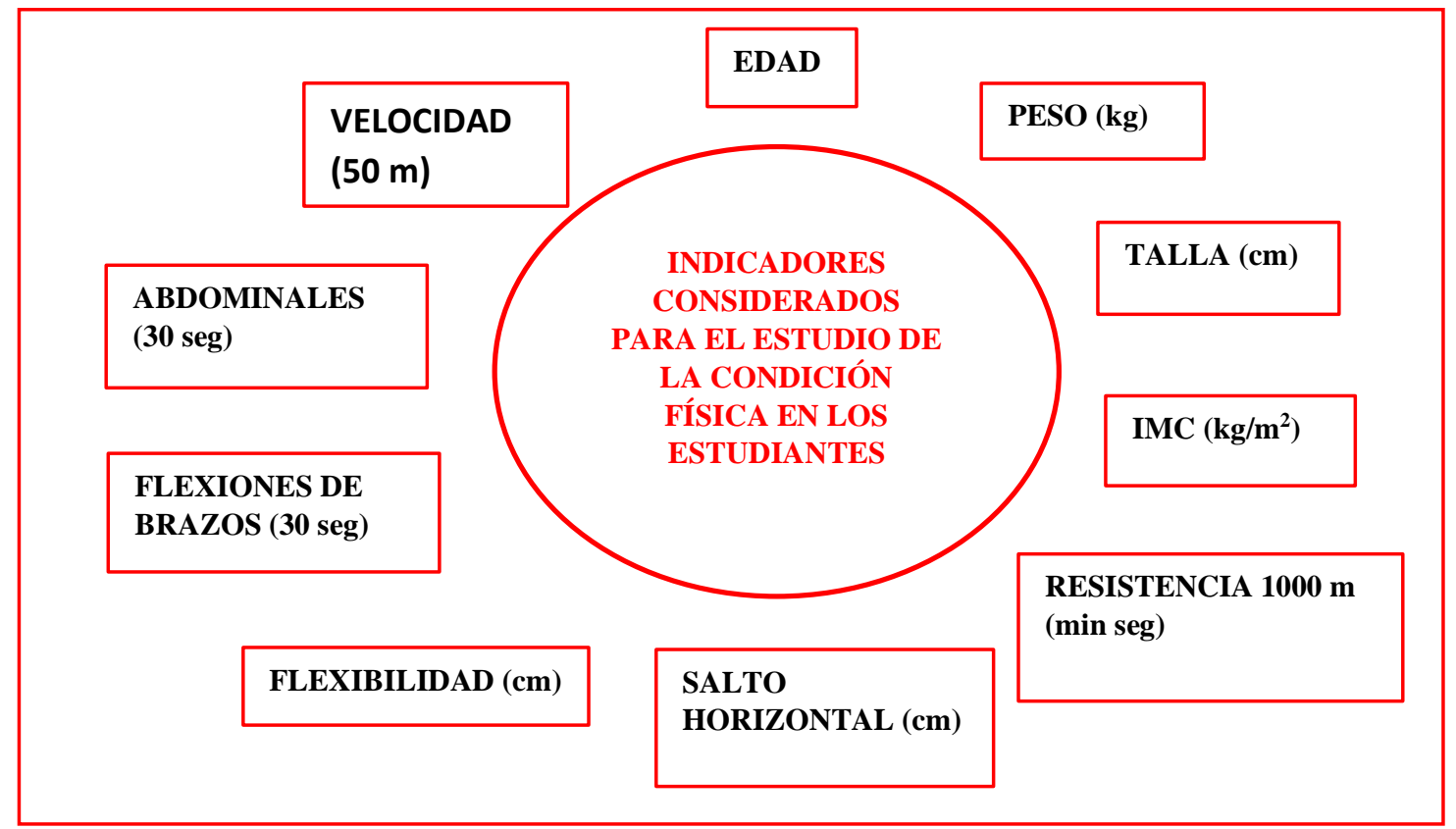

\section{Discusión de Resultados.}

Mediante los indicadores considerados para el estudio de la Condición Física de la muestra de estudiantes seleccionados, se logró valorar a un total de 1097 estudiantes, que pertenecen a 7 Facultades de la Escuela Superior del Chimborazo, propiciando un abanico de posibilidades de evaluación, en función de las Facultades a las que pertenecen.

Las Facultades son: Facultad de Ciencias (Ciencias), Facultad de Mecánica (Mecánica), Facultad de Informática y Electrónica (FIE), Facultad de Ciencias Pecuarias (Pecuarias), 
Facultad de Recursos Naturales (Recursos N), Facultad de Salud Pública y Facultad de Administración de Empresas (FADE).

A continuación, se realiza un análisis del comportamiento de la condición física de los estudiantes que conforman la muestra, en relación a los aspectos anteriormente citados.

Tabla No 1. Valoración de la Condición Física por Facultades.

\begin{tabular}{|c|c|c|c|c|c|c|c|c|c|c|c|}
\hline \multicolumn{12}{|c|}{ VALORACION DE LA CONDICION FISICA POR FACULTADES } \\
\hline FACULTAD & $\begin{array}{l}\text { Velocidad } \\
\text { En } 50 \mathrm{~m} \\
(\mathrm{se})\end{array}$ & $\begin{array}{c}\text { Abdominales } \\
\text { (cantidad de } \\
\text { repeticiones } \\
\text { en } 30 \mathrm{seg} \text { ) }\end{array}$ & $\begin{array}{c}\text { Flexibilidad } \\
\text { de brazos } \\
\text { (número de } \\
\text { repeticiones } \\
\text { En } 30 \text { seg) } \\
\end{array}$ & $\begin{array}{c}\text { Flexibilidad } \\
(\mathrm{cm})\end{array}$ & $\begin{array}{c}\text { Salto } \\
\text { horizontal } \\
(\mathrm{cm})\end{array}$ & $\begin{array}{c}\text { Resistencia } \\
\text { A } 1000 \mathrm{~m} \\
(\text { en min y seg) }\end{array}$ & $\begin{array}{l}\text { Edad } \\
\text { (años) }\end{array}$ & $\begin{array}{c}\text { Peso } \\
(k g)\end{array}$ & $\begin{array}{l}\text { Talla } \\
(\mathbf{c m})\end{array}$ & IMC & Clasificación \\
\hline CIENCIAS & 8,65 & 20,62 & 19,40 & 6,08 & 171,12 & 5,42 & 19,27 & 58,31 & 159,72 & 22,81 & NORMAL \\
\hline FADE & 9,29 & 20,35 & 21,72 & 4,87 & 154,77 & 6,09 & 19,39 & 59,86 & 159,68 & 23,43 & NORMAL \\
\hline FIE & 8,53 & 21,38 & 21,19 & 5,47 & 167,56 & 5,97 & 19,72 & 61,19 & 163,41 & 22,87 & NORMAL \\
\hline MECANICA & 7,65 & 22,19 & 25,51 & 6,30 & 197,64 & 4,84 & 20,80 & 66,10 & 166,90 & 23,67 & NORMAL \\
\hline PECUARIAS & 9,09 & 19,10 & 22,57 & 5,53 & 158,45 & 5,77 & 19,36 & 58,15 & 159,42 & 22,81 & NORMAL \\
\hline RECURSOS N & 9,23 & 18,49 & 18,23 & 5,79 & 155,96 & 5,68 & 19,58 & 60,55 & 158,34 & 24,08 & NORMAL \\
\hline $\begin{array}{l}\text { SALUD } \\
\text { PÚBLICA }\end{array}$ & 9,02 & 20,67 & 22,16 & 7,11 & 166,01 & 6,02 & 20,06 & 62,21 & 161,38 & 24,34 & NORMAL \\
\hline
\end{tabular}

Figura No 2 - Valoración de la Condición Física por Facultad.

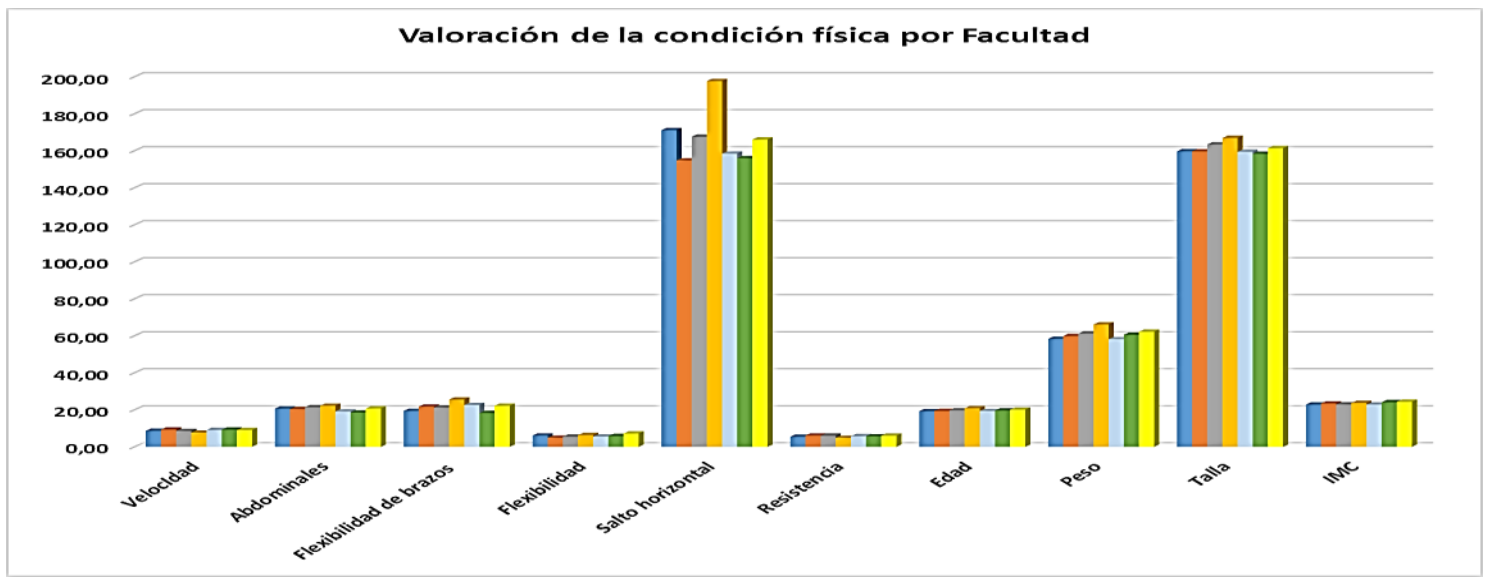

En primer lugar, se procedió a realizar el análisis de los distintos parámetros que conforman el estado de la condición física en relación a los estudiantes de las diferentes Facultades, pudiéndose constatar los siguientes resultados:

- En relación a la condición física de Velocidad en $50 \mathrm{~m}$, se pudo constatar que la velocidad promedio fue de 8,81 seg, por lo que las Facultades de FADE (9,29 seg), Pecuaria $(9,09 \mathrm{seg})$, Recursos Naturales $(9,23 \mathrm{seg})$ y Salud Pública $(9,02$ seg), obtuvieron comportamientos por debajo de la media. Es de destacar la Facultad de Mecánica, dado que sus estudiantes recorrieron los $50 \mathrm{~m}$ en 7,85 seg, lo que representa $0,96 \mathrm{seg}$ por encima de la media. 
Figura No 3 - Valoración de la Condición Física: Velocidad, por Facultades.

\begin{tabular}{|c|c|}
\hline FACULTAD & Velocldad \\
\hline CIENCIAS & 8,65 \\
\hline FADE & 9,29 \\
\hline FIE & 8,53 \\
\hline MECANICA & 7,85 \\
\hline PECUARIAS & 9,09 \\
\hline RECURSOS N & 9,23 \\
\hline $\begin{array}{c}\text { SALUD } \\
\text { PÚBLICA }\end{array}$ & 9,02 \\
\hline
\end{tabular}

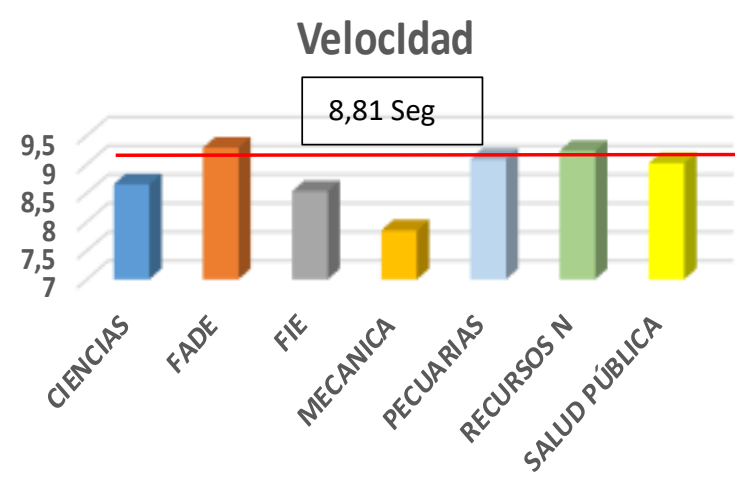

- Respecto a la condición física en el indicador de Abdominales, la media realizada fue de 20,40 repeticiones en $30 \mathrm{seg}$, resultando que en 3 Facultades: FADE $(20,35)$, Pecuarias $(19,10)$ y Recursos Naturales $(18,49)$, los estudiantes quedaron por debajo de la media. Se destaca la Facultad de Mecánica, al realizar sus estudiantes 22,19 repeticiones en $30 \mathrm{seg}$, representando 1,79 repeticiones por encima de la media.

Figura No. 4- Valoración de la Condición Física: Abdominales, por Facultades.

\begin{tabular}{|c|c|}
\hline FACULTAD & Abdominales \\
\hline CIENCIAS & 20,62 \\
\hline FADE & 20,35 \\
\hline FIE & 21,38 \\
\hline MECANICA & 22,19 \\
\hline PECUARIAS & 19,1 \\
\hline RECURSOS N & 18,49 \\
\hline $\begin{array}{c}\text { SALUD } \\
\text { PÚBLICA }\end{array}$ & 20,67 \\
\hline
\end{tabular}

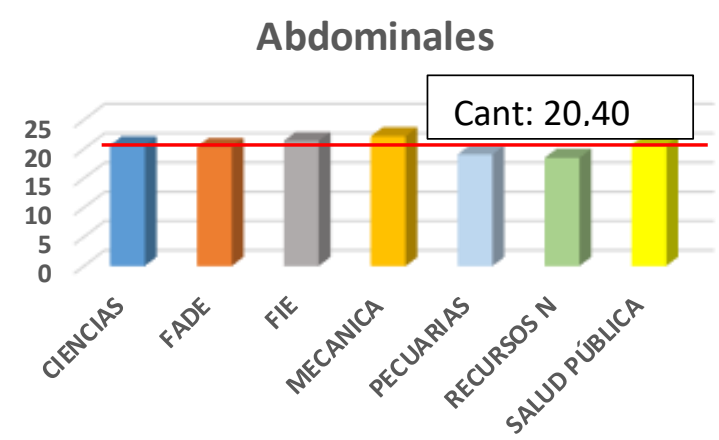

- Relacionado con la condición física en el indicador flexibilidad de brazos en un tiempo de 30 segundos, se obtuvo una media de 21,54, ello implica que 3 facultades: Ciencias $(19,4)$, FIE $(21,19)$ y Recursos Naturales $(18,23)$ quedaron por debajo de la media, destacándose la Facultad de Mecánica, al lograr sus estudiantes un número de 25,51, o sea 3,97 flexiones más, por encima de la media. 
Figura No 5- Valoración de la Condición Física: Flexibilidad de brazos, por Facultades.

\begin{tabular}{|c|c|}
\hline FACULTAD & $\begin{array}{c}\text { Flexibilidad } \\
\text { de brazos }\end{array}$ \\
\hline CIENCIAS & 19,4 \\
\hline FADE & 21,72 \\
\hline FIE & 21,19 \\
\hline MECANICA & 25,51 \\
\hline PECUARIAS & 22,57 \\
\hline RECURSOS N & 18,23 \\
\hline $\begin{array}{c}\text { SALUD } \\
\text { PÚBLICA }\end{array}$ & 22,16 \\
\hline
\end{tabular}

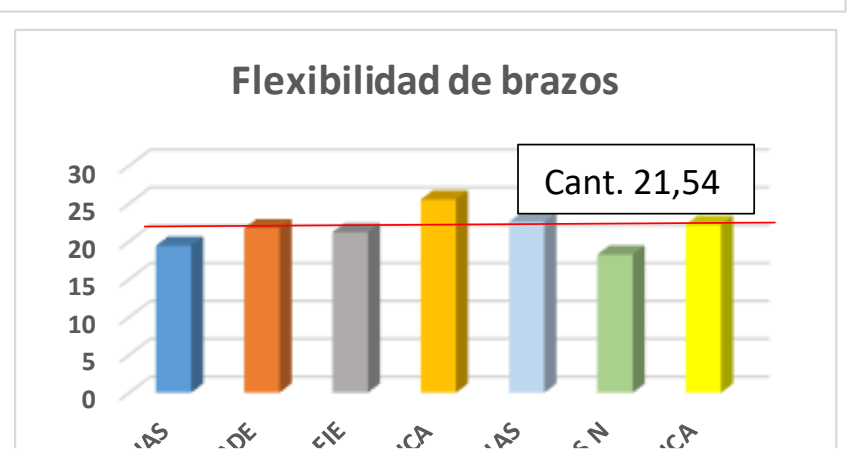

- En relación a la condición física de flexibilidad, la media obtenida fue de 5,88 cm, resultando que 4 facultades: FADE $(4,87 \mathrm{~cm}), \operatorname{FIE}(5,47 \mathrm{~cm})$, Pecuarias $(5,53 \mathrm{~cm})$ y Recursos Naturales $(5,79 \mathrm{~cm})$, quedaron por debajo de la media. Destaca la Facultad de Salud Pública cuyos estudiantes lograron $7,11 \mathrm{~cm}$, o sea 1,23 cm por encima de la media.

Figura No 6- Valoración de la Condición Física: Flexibilidad, por Facultades.

\begin{tabular}{|c|c|}
\hline FACULTAD & Flexibilidad \\
\hline CIENCIAS & 6,08 \\
\hline FADE & 4,87 \\
\hline FIE & 5,47 \\
\hline MECANICA & 6,3 \\
\hline PECUARIAS & 5,53 \\
\hline $\begin{array}{c}\text { RECURSOS } \\
\text { NATURALES }\end{array}$ & 5,79 \\
\hline $\begin{array}{c}\text { SALUD } \\
\text { PÚBLICA }\end{array}$ & 7,11 \\
\hline
\end{tabular}

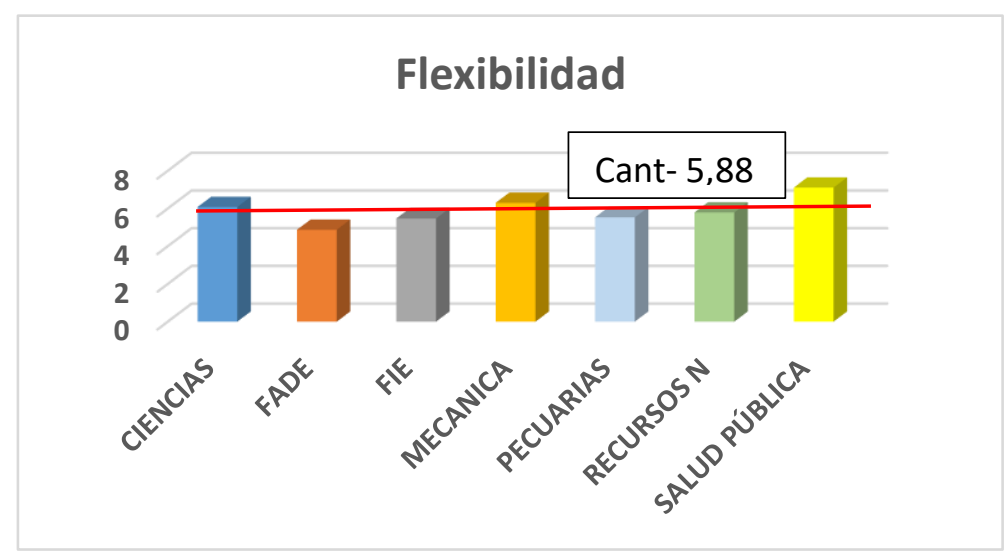

- Respecto a la condición física de salto horizontal, se logró una media de 167,36 $\mathrm{cm}$, por lo que 4 facultades: FADE $(154,77 \mathrm{~cm})$, Pecuarias $(158,45 \mathrm{~cm})$, Recursos Naturales $(155,96 \mathrm{~cm})$ y Salud Pública $(166,01 \mathrm{~cm})$, quedaron por debajo de la media. Se destaca la Facultad de Mecánica, al lograr sus estudiantes una longitud de salto de 197,64 cm, o sea 30,28 cm por encima de la media. 
Figura No 7- Valoración de la Condición Física: Salto horizontal, por Facultades.

\begin{tabular}{|c|c|}
\hline FACULTAD & $\begin{array}{c}\text { Salto } \\
\text { horizontal }\end{array}$ \\
\hline CIENCIAS & 171,12 \\
\hline FADE & 154,77 \\
\hline FIE & 167,56 \\
\hline MECANICA & 197,64 \\
\hline PECUARIAS & 158,45 \\
\hline RECURSOS N & 155,96 \\
\hline $\begin{array}{c}\text { SALUD } \\
\text { PÚBLICA }\end{array}$ & 166,01 \\
\hline
\end{tabular}

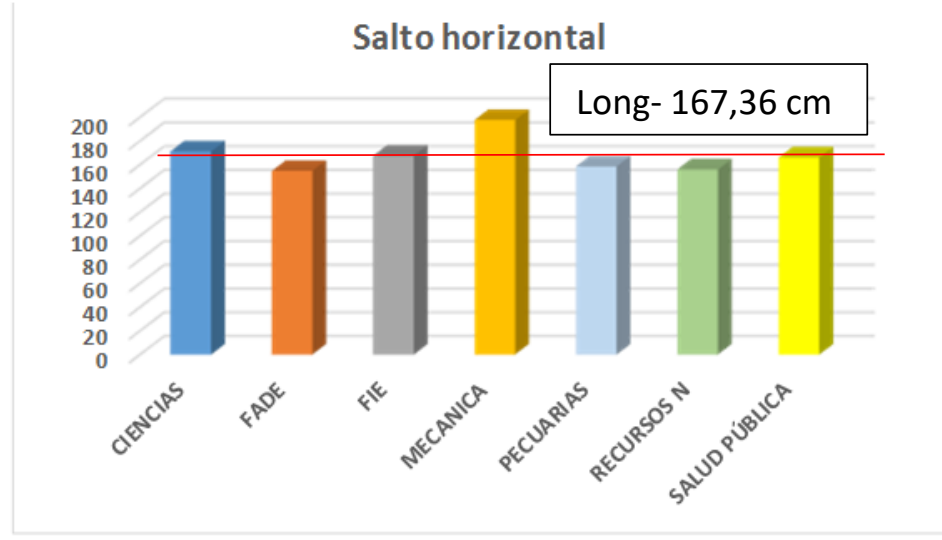

- Relacionado con la condición física de resistencia a $1000 \mathrm{~m}$, se obtuvo una media de 5 min 68 seg, de ello resulta que 4 facultades: FADE (6 min 09 seg), FIE (5 min $97 \mathrm{seg}$ ), Pecuarias (5 min $77 \mathrm{seg}$ ) y Salud Pública (6 min 2 seg), lograron tiempos por encima de la media. Es de destacar la Facultad de Mecánica, cuyos estudiantes lograron recorrer la distancia en $4 \mathrm{~min} 84 \mathrm{seg}$, lo que representa 0,84 seg por debajo de la media.

Figura No 8- Valoración de la Condición Física: Resistencia, por Facultades.

\begin{tabular}{|c|c|}
\hline FACULTAD & Resistencia \\
\hline CIENCIAS & 5,42 \\
\hline FADE & 6,09 \\
\hline FIE & 5,97 \\
\hline MECANICA & 4,84 \\
\hline PECUARIAS & 5,77 \\
\hline RECURSOS N & 5,68 \\
\hline $\begin{array}{c}\text { SALUD } \\
\text { PÚBLICA }\end{array}$ & 6,02 \\
\hline
\end{tabular}

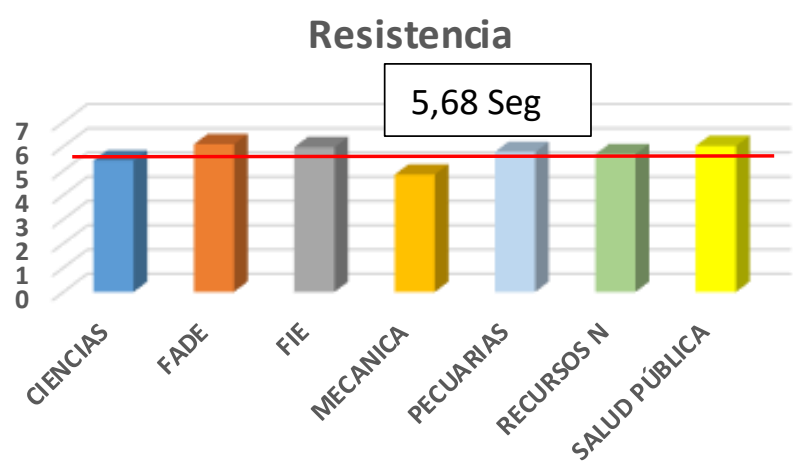

El análisis realizado dio como resultado que, entre las 7 facultades, los estudiantes de la Facultad de Mecánica, son los que presentan una mejor condición física, lo cual se expone en el siguiente gráfico: 
Figura No 9- Comportamiento de la Condición Física por Facultades

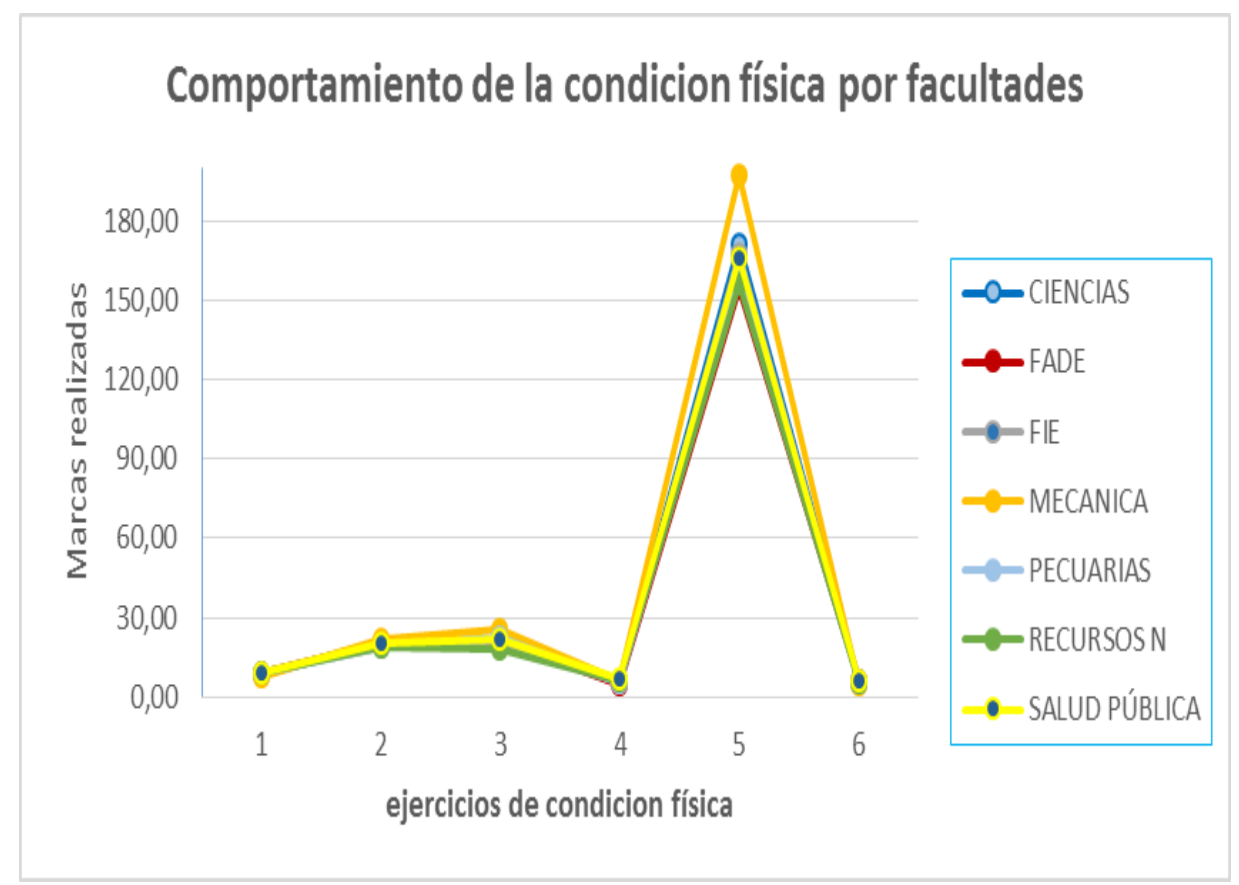

Los estudiantes de la Facultad de Mecánica, fueron superiores en las diferentes pruebas realizadas, logrando marcas por encima del promedio entre todas las facultades.

Figura No 10- Desempeño de la Condición Física de la Facultad de Mecánica

\begin{tabular}{|l|c|c|c|c|c|c|}
\hline & Velocldad & Abdominales & $\begin{array}{c}\text { Flexibilidad } \\
\text { de brazos }\end{array}$ & Flexibilidad & $\begin{array}{c}\text { Salto } \\
\text { horizontal }\end{array}$ & Resistencia \\
\hline MECANICA & 7,65 & 22,19 & 25,51 & 6,30 & 197,64 & 4,84 \\
\hline PROMEDIO & 8,81 & 20,4 & 21,54 & 5,88 & 167,36 & 5,68 \\
\hline
\end{tabular}

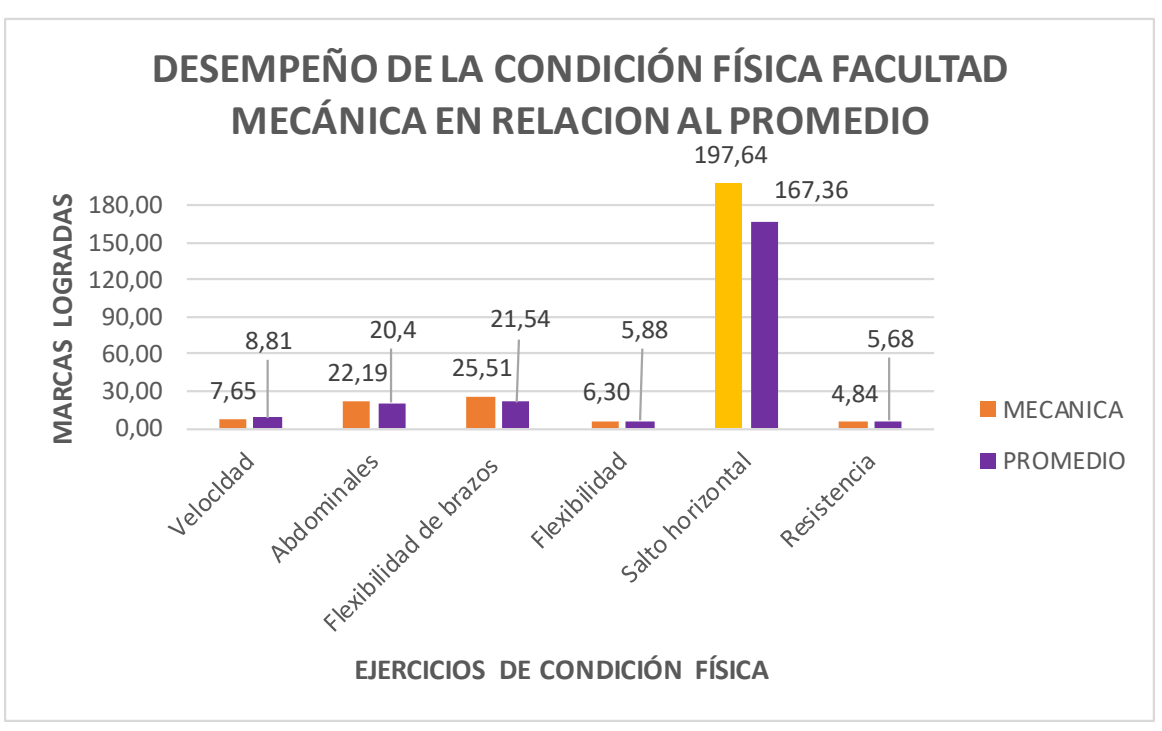


De igual manera se evaluaron también los indicadores de talla y peso en relación a la edad de los estudiantes de las diferentes facultades, con el objetivo de medir el Índice de Masa Corporal (IMC) de los mismos.

- En relación a la edad de los estudiantes de las diferentes facultades, la media fue de 19,74 años. En 5 de las distintas facultades: Ciencias (19,27 años), FADE (19,39 años), FIE (19,72 años), Pecuarias (19,36 años) y Recursos Naturales (19,58 años), los estudiantes presentan un promedio de edad inferior a la media. Se remarca la Facultad de Mecánica, en la que los estudiantes promedian una edad de 20,80 años, o sea 1 mes y 6 días superior a la media.

Figura No 11- Valoración de la Edad de los estudiantes, por Facultades.

\begin{tabular}{|c|c|}
\hline FACULTAD & Edad \\
\hline CIENCIAS & 19,27 \\
\hline FADE & 19,39 \\
\hline FIE & 19,72 \\
\hline MECANICA & 20,8 \\
\hline PECUARIAS & 19,36 \\
\hline RECURSOS N & 19,58 \\
\hline $\begin{array}{c}\text { SALUD } \\
\text { PÚBLICA }\end{array}$ & 20,06 \\
\hline
\end{tabular}

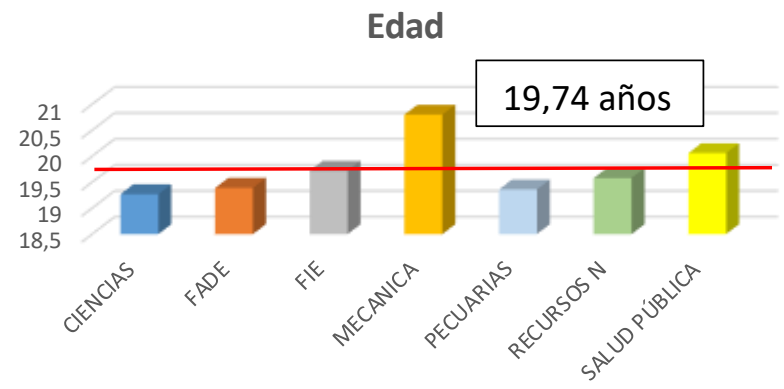

- En relación al peso de los estudiantes de las diferentes facultades, la media fue de $60,91 \mathrm{~kg}$, por lo que 4 facultades: Ciencias $(58,31 \mathrm{Kg})$, FADE $(59,86 \mathrm{Kg})$, Pecuarias $(58,15 \mathrm{~kg})$ y Recursos Naturales $(60,55 \mathrm{~kg})$ presentan un peso menor a la media. Se destaca la Facultad de Mecánica, donde el promedio de peso de los estudiantes es de $66,10 \mathrm{~kg}$, o sea $5,19 \mathrm{~kg}$ superior a la media

Figura No 12- Valoración del Peso de los estudiantes, por Facultades.

\begin{tabular}{|c|c|}
\hline FACULTAD & Peso \\
\hline CIENCIAS & 58,31 \\
\hline FADE & 59,86 \\
\hline FIE & 61,19 \\
\hline MECANICA & 66,1 \\
\hline PECUARIAS & 58,15 \\
\hline RECURSOS N & 60,55 \\
\hline $\begin{array}{c}\text { SALUD } \\
\text { PÚBLICA }\end{array}$ & 62,21 \\
\hline
\end{tabular}

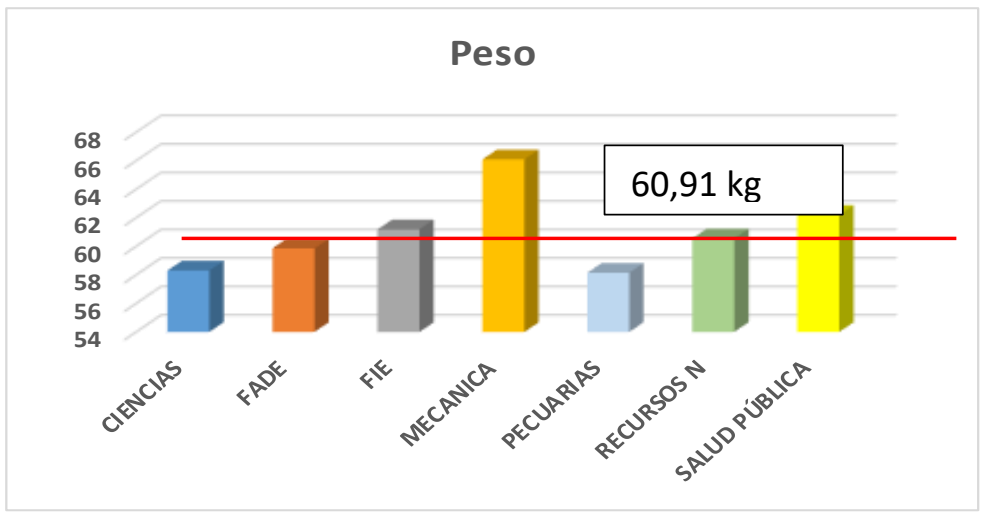

- Respecto a la Talla de los estudiantes de las facultades, la media fue de 161,26 cm, encontrándose 4 facultades: Ciencias $(159,72 \mathrm{~cm})$, FADE $(159,68 \mathrm{~cm})$, Pecuarias $(159,42 \mathrm{~cm})$ y Recursos Naturales $(158,34 \mathrm{~cm})$. Se destaca la Facultad 
de Mecánica, cuyos estudiantes presentan un promedio de $166,90 \mathrm{~cm}$, superior en $5,64 \mathrm{~cm}$.

Figura No 13- Valoración de la Talla de los estudiantes, por Facultades.

\begin{tabular}{|c|c|}
\hline FACULTAD & Talla \\
\hline CIENCIAS & 159,72 \\
\hline FADE & 159,68 \\
\hline FIE & 163,41 \\
\hline MECANICA & 166,9 \\
\hline PECUARIAS & 159,42 \\
\hline RECURSOS N & 158,34 \\
\hline $\begin{array}{c}\text { SALUD } \\
\text { PÚBLICA }\end{array}$ & 161,38 \\
\hline
\end{tabular}

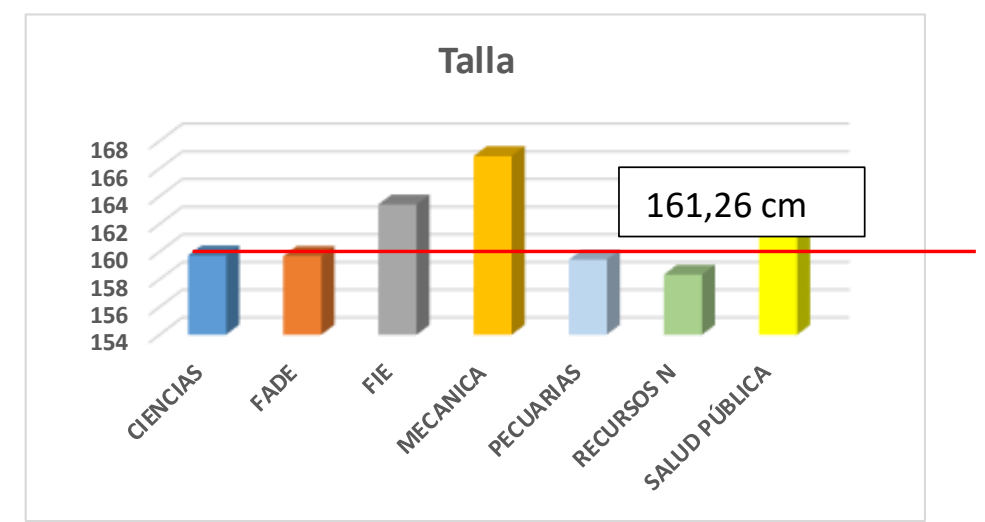

- En relación con el Índice de Masa Corporal (IMC) de los estudiantes de las facultades, la media fue de 23,43, considerándose como Normal. Se constató que resultaron 3 facultades: Ciencias $(22,81)$, Fie $(22,87)$ y Pecuarias $(22,81)$, que presentaron un IMC inferior a la media. De igual manera la Facultad de Salud Pública, presentó un $\mathrm{IMC}=24,34$, cifra que se encuentra muy cerca del límite de sobrepeso $(25,0)$.

Figura No 14- Valoración de IMC de los estudiantes, por Facultades.

\begin{tabular}{|c|c|}
\hline FACULTAD & IMC \\
\hline CIENCIAS & 22,81 \\
\hline FADE & 23,43 \\
\hline FIE & 22,87 \\
\hline MECANICA & 23,67 \\
\hline PECUARIAS & 22,81 \\
\hline RECURSOS N & 24,08 \\
\hline $\begin{array}{c}\text { SALUD } \\
\text { PÚBLICA }\end{array}$ & 24,34 \\
\hline
\end{tabular}

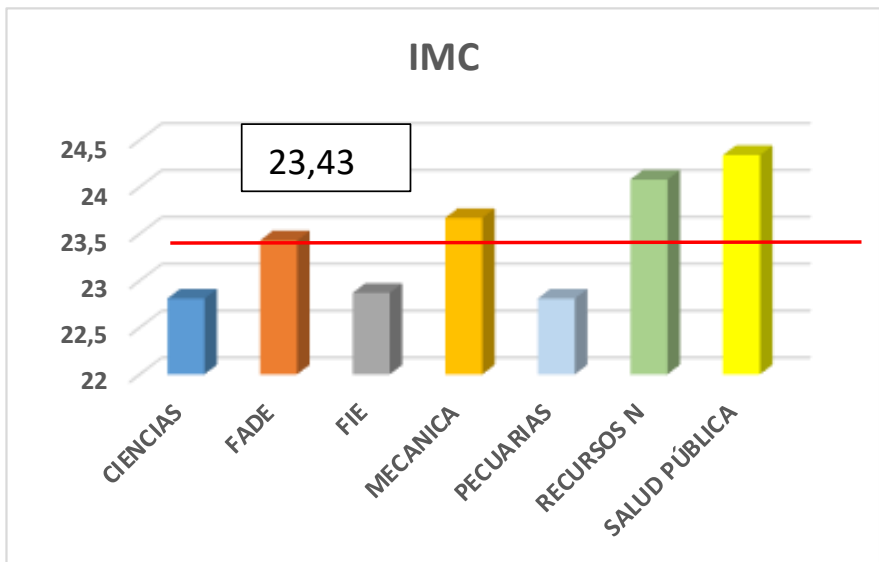

Cuando analizamos el resultado del estado de la condición física, pudimos constatar la relación existente entre los indicadores de edad-peso-talla- IMC, de los estudiantes y el desempeño de la condición física, al constatarse que los estudiantes de la Facultad de Mecánica, presentaron una edad, talla y peso superior al resto de los estudiantes del resto de las facultades. 
No obstante, también hay que tener en cuenta el indicador sexo, dado que, en el caso de la Facultad de Mecánica, la proporción del sexo femenino respecto al sexo masculino es de $9,50 \%$, o sea prevalece el sexo masculino en los estudiantes de esta facultad. En el resto de las facultades la proporción es mayor al 29,0\%.

Como puede apreciarse el estudio de la condición física en los estudiantes objeto de estudio, permite integrar dos aspectos esenciales dentro de los resultados encontrados:

1. Se aporta un sistema de indicadores adaptados a las características y condiciones de los participantes, el cual contribuyó a realizar la valoración integral del estado de la condición física de los estudiantes participantes.

2. Se reafirma la importancia y utilidad del diagnóstico oportuno sobre la condición física en los estudiantes evaluados como aspecto esencial para su desarrollo integral.

Por otra parte, se coincide en que resulta extremadamente importante estudiar la condición física desde la base del diagnóstico oportuno, estableciendo indicadores que permitan caracterizar y dar seguimiento a los resultados individuales, de modo tal que se pueda contribuir a mejorar la calidad de vida de la población estudiantil universitaria.

\section{Conclusiones.}

A modo de conclusión se considera que:

- El estudio de la condición física, realizado de manera oportuna en la población estudiantil universitaria, resulta de mucha utilidad. El mismo propicia, mediante el reconocimiento de los indicadores de evaluación, establecer estrategias de intervención encaminadas a mejorar la calidad de vida de la población estudiantil universitaria y aportar soluciones viables para la prevención de posibles enfermedades y la optimización del desempeño individual de cada estudiante.

\section{Referencias Bibliográficas}

Arbós, MT (2017) Actividad Física y Salud en Estudiantes Universitarios desde una perspectiva Salutogénica Berenguer. Tesis Doctoral Universitat de les Illes Balears.

Gálvez A, Rodríguez P, Guillamón A, García-Cantó E, Pérez J, Loreto M y Tárraga P, (2015)- Nivel de condición física y su relación con el estatus de peso corporal en escolares. Revista Nutrición Hospitalaria. Nutr Hosp. 2015;31(1):393-400 ISSN 0212-1611 • CODEN NUHOEQ S.V.R. 318. DOI:10.3305/nh.2015.31.1.8074.

González Sandoval CE, Díaz Burke Y, Mendizabal-Ruiz AP, Medina Díaz E, Morales JA. (2017). Prevalencia de obesidad y perfil lipídico alterado en jóvenes universitarios. Nutr Hosp. 2017;29(2):315-21. DOI:10.3305/nh.2014.29.2.7054.

Guillamón, R. (2018). Análisis de la relación entre salud, ejercicio físico y condición física en escolares y adolescentes. Revista Ciencias de la Actividad Física UCM, $\mathrm{N}^{\circ}$ 20(1) enero-junio, 1-15. DOI: http://doi.org/10.29035/rcaf.20.1.1 
Laguado J, Gómez, MP., (2014). Estilos de vida saludable en estudiantes de enfermería en la Universidad Cooperativa de Colombia. Revista Hacia la Promoción de la Salud, Vol. 19, Núm. 1, pp. 68-83 Universidad de Caldas.

Martínez, L., (2008). Teoría y Praxis Investigativa, Volumen 3 - No. 1. Centro de Investigación y Desarrollo • CID / Fundación Universitaria del Área Andina.

Organización Panamericana de la Salud (OPS) (2019). Plan de Acción Mundial sobre Actividad Física 2018-2030.Mas personas activas para un mundo sano ISBN: 978-92-75-32060-0; https://creativecommons.org/licenses/by-nc-sa/3.0/igo).

Ruiz JR, Castro-Pinero J, Artero EG, Ortega FB, Sjostrom M, Suni J, Castillo MJ. Predictive validity of health-related fitness in youth: a systematic review. $\mathrm{Br} \mathbf{J}$ Sports Med 2009; 43(12): 909-923.

Rush E, McLennan S, Obolonkin V, Vandal, AC, Hamlin M, Simmons D, Graham D. (2014). Project Energize: whole-region primary school nutrition and physical activity programme; evaluation of body size and fitness 5 years after the randomised controlled trial. Br J Nutr 2014; 111 (2): 363-371.

Valdés, P., Godoy, A., Herrera, T., -,4 y Duran, S., (2015) Revista Nutrición Hospitalaria. 32(2):829-836 CODEN NUHOEQ S.V.R. 318. DOI:10.3305/nh.2015.32.2.9194

Vásquez-Gómez, Jaime A; Castillo-Retamal, Marcelo E; Souza de Carvalho, Ricardo; Faundez-Casanova, Cesar P; Torrealba-Campos, Angélica P, (2018)Antropometría, nivel de actividad física y condición física en estudiantes de educación física tras cuatro años en la universidad, Revista nutrición clínica y Dietética Hospitalaria. Nutr. clín. diet. hosp. 2018; 38(1):160-164 DOI: $10.12873 / 381$.

Zenić N, Foretić N, Blazević M. Nonlinear relationships between anthropometric and physical fitness variables in untrained pubescent boys. Coll Antropol 2013; 37 (2): 153-9.

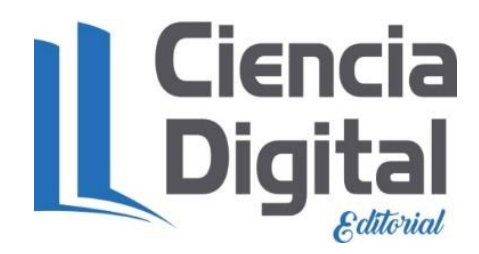




\section{PARA CITAR EL ARTÍCULO INDEXADO.}

Díaz, L. G., Lomas Badillo, P. L., Tocto Lobato, J. G., \& Sánchez Espinoza, O. F. (2021). Valoración integral de la condición física en estudiantes de la ESPOCH: Un diagnóstico oportuno. ConcienciaDigital, 4(1.2), 390-404. https://doi.org/10.33262/concienciadigital.v4i1.2.1605

\section{\Ciencia}

El artículo que se publica es de exclusiva responsabilidad de los autores y no necesariamente reflejan el pensamiento de la Revista Conciencia Digital.

El artículo queda en propiedad de la revista y, por tanto, su publicación parcial y/o total en otro medio tiene que ser autorizado por el director de la Revista Conciencia Digital.

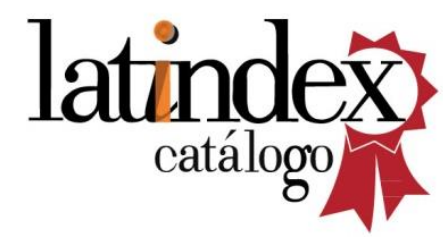

